\title{
The Prognostic Value of Cancer Stem Cell Markers in Cervical Cancer: A Systematic Review and Meta-Analysis
}

\author{
Moh Nailul Fahmi ${ }^{1,2}$, Inge Nandya Hertapanndika ${ }^{3}$, Fitriyadi Kusuma ${ }^{4 *}$
}

\begin{abstract}
Objectives: Prognostic biomarkers in cervical cancer are widely investigated, including cancer stem cell (CSC) markers. However, their significance remains uncertain. This study aimed to determine the role of cervical cancer stem cell (CCSC) markers for survival. Materials and Methods: We conducted a systematic review and meta-analysis (PROSPERO CRD42021237072) of studies reporting CCSC markers as the prognostic predictor based on PRISMA guidelines. We included English articles investigating associations of CCSCs expression in tissue tumor with overall survival (OS) or disease-free survival (DFS) from PubMed, EBSCO, and The Cochrane Library databases. The quality of studies was analyzed based on Newcastle-Ottawa Quality Assessment Scale. Results: From 413 publications, after study selection with inclusion and exclusion criteria, 22 studies were included. High expressions of CCSC markers were associated with poor OS and DFS ( $\mathrm{HR}=1.05,95 \% \mathrm{CI}: 1.03-1.07, \mathrm{P}<0.0001$; HR=1.31, 95\% CI: $1.09-1.17$, $\mathrm{P}<0.00001$; respectively). Sub-analysis of individual CCSC markers indicated significant correlations between CD44 $(\mathrm{HR}=1.14,95 \% \mathrm{CI}: 1.07-1.22, \mathrm{P} 0.0001), \mathrm{SOX} 2(\mathrm{HR}=1.58,95 \% \mathrm{CI}: 1.17-2.14, \mathrm{P} 0.003), \mathrm{OCT} 4(\mathrm{HR}=1.03$, 95\% CI: $1.01-1.06$, P 0.008), ALDH1 (HR=1.36, 95\% CI: $1.13-1.64, \mathrm{P} 0.001)$, and CD49f (HR=3.02, 95\% CI: $1.37-6.64, \mathrm{P} 0.006)$ with worse OS; OCT4 (HR=1.14, 95\% CI $1.06-1.22, \mathrm{P} 0.0003)$, SOX2 (HR=1.11, $95 \% \mathrm{CI}$ : $1.06-1.16, \mathrm{P}<0.0001)$, and ALDH1 (HR=1.22, 95\% CI: $1.10-1.35, \mathrm{P} 0.0002)$ with poor DFS. We did not conduct a meta-analysis for MSI-1 and CK17 because only one study investigated those markers. Conclusion: Expressions of OCT4, SOX2, and ALDH1 were associated with poor OS and DFS in cervical cancer tissue. These markers might have potential roles as prognostic biomarkers to predict unfavorable survival.
\end{abstract}

Keywords: Cervical cancer stem cell marker- prognostic biomarker- OCT4- SOX2- ALDH1

Asian Pac J Cancer Prev, 22 (12), 4057-4065

\section{Introduction}

Cervical cancer is the fourth most common type of gynecological cancer worldwide (Pimple et al., 2016). Most cervical cancer is caused by human papillomavirus (HPV) infection (Hashiguchi et al., 2019). There were 528,000 new cases every year, with 266,000 deaths in 2012 worldwide. In developing countries, cervical cancer also has high mortality rates among women (Pimple et al., 2016). There were 14,480 new cases in the United States in 2021 (American Cancer Society, 2021). (American Cancer Society, 2021). The 5-year survival rate of cervical cancer for stage IB-IVA was $62.6 \%$ (Yoon et al., 2015).

Recently, there has been increased research on cervical cancer stem cell (CCSC) markers. The role of these markers in cancer development has been extensively highlighted. Cancer stem cells (CSCs) are a small subpopulation of tumor cells with self-renewal capabilities. There are several recognized (CSC) markers, such as CD44, aldehyde dehydrogenase 1 (ALDH1), OCT4, and SOX2 (Liu H et al., 2016; López et al., 2012; Ortiz-Sánchez et al., 2016). These markers are thought to play a role in the progression, development, recurrence, and survival of cancer (Dalerba et al., 2007). These CSC markers are also identified to have the potential for tumorigenesis, including spherogenesis, resistance to cytotoxic drugs, and ionizing radiation (Dobbin and Landen, 2013). Therefore, the assessment of CSC markers is helpful for preventing metastasis and cervical cancer recurrence (Muralikrishnan et al., 2020).

The prognostic biomarkers have an important value to guide cancer therapy. Many prognostic factors and predictive markers have been studied. Prognostic factors for cervical cancer include lymph node status, tumor size,

${ }^{1}$ Department of Obstetrics and Gynecology, Faculty of Medicine, Public Health and Nursing, Universitas Gadjah Mada, Dr. Sardjito Hospital, Yogyakarta, Indonesia. ${ }^{2}$ Fellowship Gynecology Oncology Division, Department of Obstetrics and Gynecology, Universitas Indonesia, Dr. Cipto Mangunkusumo General Hospital, Faculty of Medicine, Jakarta, Indonesia. ${ }^{3}$ Dr. Sayidiman Regional Public Hospital, Magetan, East Java, Indonesia. ${ }^{4}$ Gynecology Oncology Division, Department of Obstetrics and Gynecology, Universitas Indonesia, Dr. Cipto Mangunkusumo General Hospital, Faculty of Medicine, Jakarta, Indonesia. *For Correspondence: fitriyadikusuma@gmail.com 
invasion of the parametrium tissue, depth of invasion, presence or absence of lymphovascular space invasion, histology, grading, stromal reactions, and age. The accuracy of these factors still depends on the variation of each individual (Rasjidi I, 2009). It is important to know the other prognostic factors in cervical cancer both from obstetric-gynecological and other medical aspects such as the identification of stem cell markers.

Several markers of CCSC have been identified, such as CD44, SOX2, ALDH, OCT4, and CD49f. Some studies have found the basis for CCSC markers as a prognostic factor. However, there has been no systematic review assessing the overall role of CSCs in cervical cancer. In this study, we conducted a systematic review and meta-analysis of published studies. This study aimed to determine the role of CCSC markers in overall survival (OS) and disease-free survival (DFS).

\section{Materials and Methods}

\section{Search strategy}

The protocol of this study was registered in the International Prospective Register of Systematic Reviews (PROSPERO) with registration number CRD42021237072 and conducted in accordance with PRISMA guidelines (Moher et al., 2009). We used search terms [((cervical or cervix) and (cancer* or carcinoma* or neoplasm* or malignan* or tumor*) or ("uterine cervical neoplasm")) and (ALDH or ALDH1 or CD44 or MSI1 or OCT4 or SOX2 or CD49f or CD133 or CK17 or "ABC transporter*")]. We searched literature from PubMed, EBSCO, and The Cochrane Library from 1951 up to 2021, as shown in Figure 1.

\section{Selection criteria}

\section{Types of study}

All candidate articles were initially screened by title and/or abstract using the following inclusion criteria. We included case-control or cohort studies investigating the association between CCSC markers including aldehyde dehydrogenase-1 (ALDH1), CD44, sex determining region Y-box 2 (SOX2), octamer-binding transcription factor 4 (OCT4), Musashi 1 (MSI1), CD49f, CD133, cytokeratin 17 (CK17), ABC transporter), examined by immunohistochemistry (IHC) or polymerase chain reaction $(\mathrm{PCR})$ or enzyme-linked immunosorbent assay (ELISA) or reverse transcription-PCR (RT-PCR) or flow cytometry, and survival, both OS and DFS). Studies reporting hazard ratios (HRs) and 95\% confidence intervals (CIs), or data sufficient to estimate HR and 95\% CIs from survival analyzes; sample size $\geq 20$ cases; written in English; published in a peer-reviewed journal were the inclusion criterion. If several articles were reported by the same group, only the most complete paper was included. Non-research articles or studies that focus on animal or human cell lines or uninformed papers on the prognosis of cervical cancer were excluded.

\section{Types of participant}

The participants were cervical cancer patients diagnosed histo-pathologically. We excluded patients with co-existing primary malignancy in other organs.

Data extraction and study selection

We independently scanned article titles to exclude irrelevant studies. We reviewed abstracts from the remaining articles to find potentially relevant studies and excluded duplicate studies. The full texts of the potentially relevant studies were reviewed to be included in this study based on the eligibility criteria. We resolved disputes by discussion and, if necessary, engaged a third reviewer to make a final decision. Data drawn from the study included first author, year of publication, country, study design, CCSC marker, measurement test method, specimen type, sample size, measurement cut-off, follow-up time, and value effect. The endpoints of this study are OS and DFS.

\section{Quality assessment}

The study quality was evaluated using the NewcastleOttawa Assessment Scale (NOS). Each study was assessed by three authors independently (MNF, INH, and FK). The scale scoring uses a star system which indicates the quality of the included studies. The score of high quality was defined as $\geq 7$ stars (Appendix 1 and 2).

\section{Statistical analysis}

The MedCalc software version 19.8.0 was used by a clinical epidemiologist and biostatistician in the metaanalysis to get the value for the pooled odds ratio of the CSC markers in cervical cancer. The pooled odds ratio was with 95\% CI. Significant heterogeneity was indicated by $\mathrm{I}^{2}>50 \%$. A fixed-effect model was performed. Publication bias was evaluated by using Egger's and Begg's tests.

\section{Results}

A total of 413 publications were obtained from PubMed, EBSCO, and the Cochrane Library using our search methods. The records after removing duplicates were 319 studies. After reviewing the titles and abstracts, we excluded 109 studies for several reasons, including study design (review studies, case reports), non-English languages, using cell line, and animal study. From the number of studies, we reviewed the full text and finally included 102 studies that investigated the prognostic value of CSC markers in cervical cancer. After a deep review, 80 full-text studies were excluded for some reasons, including studies that did not assess the survival and were mixed with non-cervical cancer. Twenty-two studies matched the inclusion and exclusion criteria of this study (Appendix 3 ). All of the included studies used the tissue specimen.

Twenty-two studies analyzed the role of various CCSC markers as prognostic biomarkers of cervical cancer. The studies were conducted in European countries ( 7 studies), Asian countries (13 studies), American countries (1 study), and Oceanian countries (1 study). Ten studies investigated CD44 expression, 7 studies looked at SOX2 expression, 5 studies assessed OCT4 expression, 5 studies looked at ALDH1 expression, and 2 studies looked at CD49f. Meanwhile, only 1 study assessed both MSI1 and CK17 expressions (Appendix 4).

Based on NOS, there were 13 studies which have high quality study, including Kim et al., 2015; Hellberg et al., 
2009; Ayhan et al., 2001; Speiser et al., 1999; Costa et al., 2001; Shen et al., 2014; Yang Y et al., 2014; Yao et al., 2014; Lv et al., 2015; Fu et al., 2018; Hou et al., 2015; Xie et al., 2016; and Hashiguchi et al., 2019. Meanwhile, 9 studies have low quality study, including Chopra et al., 2018; Lambaudie et al., 2014; Uhl-Steidl et al., 1998; Kainz et al., 1995; Speiser et al., 1997; Yang Z et al., 2014; Kainz et al., 1996; Ji et al., 2014; and Ammothumkandy et al., 2016.

\section{Study design}

Twenty-two studies investigated 7 markers of CSCs (CD44, SOX2, OCT4, ALDH1, MSI-1, CD49f, CK17) in cervical cancer. All of the studies used cohort design to assess the prognostic values of each of the CCSC markers, and three of them were retrospective studies.

Eleven studies investigated all stages of cervical cancer (Chopra et al., 2018; Kim et al., 2015; Hellberg et al., 2008; Lambaudie et al., 2014; Uhl-Steidl et al., 1998; Kainz et al., 1995; Kainz et al., 1996; Ji et al., 2014; Shen et al., 2014; Lv et al., 2015; Hashiguchi et al., 2019; Ammothumkandy et al., 2016). Meanwhile, 10 studies only assessed the early stages of cervical cancer (Ayhan et al., 2001; Speiser et al., 1999; Speiser et al., 1997; Yang Z et al., 2014; Costa et al., 2001; Yang Y et al., 2014; Yao et al., 2014; Fu et al., 2018; Hou et al.,
2015; Xie et al., 2016).

\section{Sample size}

The sample size was varied from 31 up to 630 participants among the studies. The total number of participants in this review was 3,930 from 22 studies. Fifteen studies included only patients with cervical cancer. The rest of them involved either normal or benign cervical intraepithelial lesions as control groups.

\section{Measurement methods}

All twenty-one included studies used immunohistochemistry staining on paraffin-embedded samples from tissue tumors. The staining of the studies used quantitative, qualitative, or semi-quantitative scoring methods. The scoring system was varied between all of the studies. There was only one study (Ammothumkandy et al., 2016) that examined CCSC marker using flow cytometry.

\section{Association between cervical cancer stem cell (CCSC)} markers expression and overall survival (OS)

In our study, there were 8 studies (2,201 patients) that reported adequate information to evaluate the correlation between CSC markers and OS in cervical cancer patients, as shown in Figure 2. Overexpression of overall CCSC
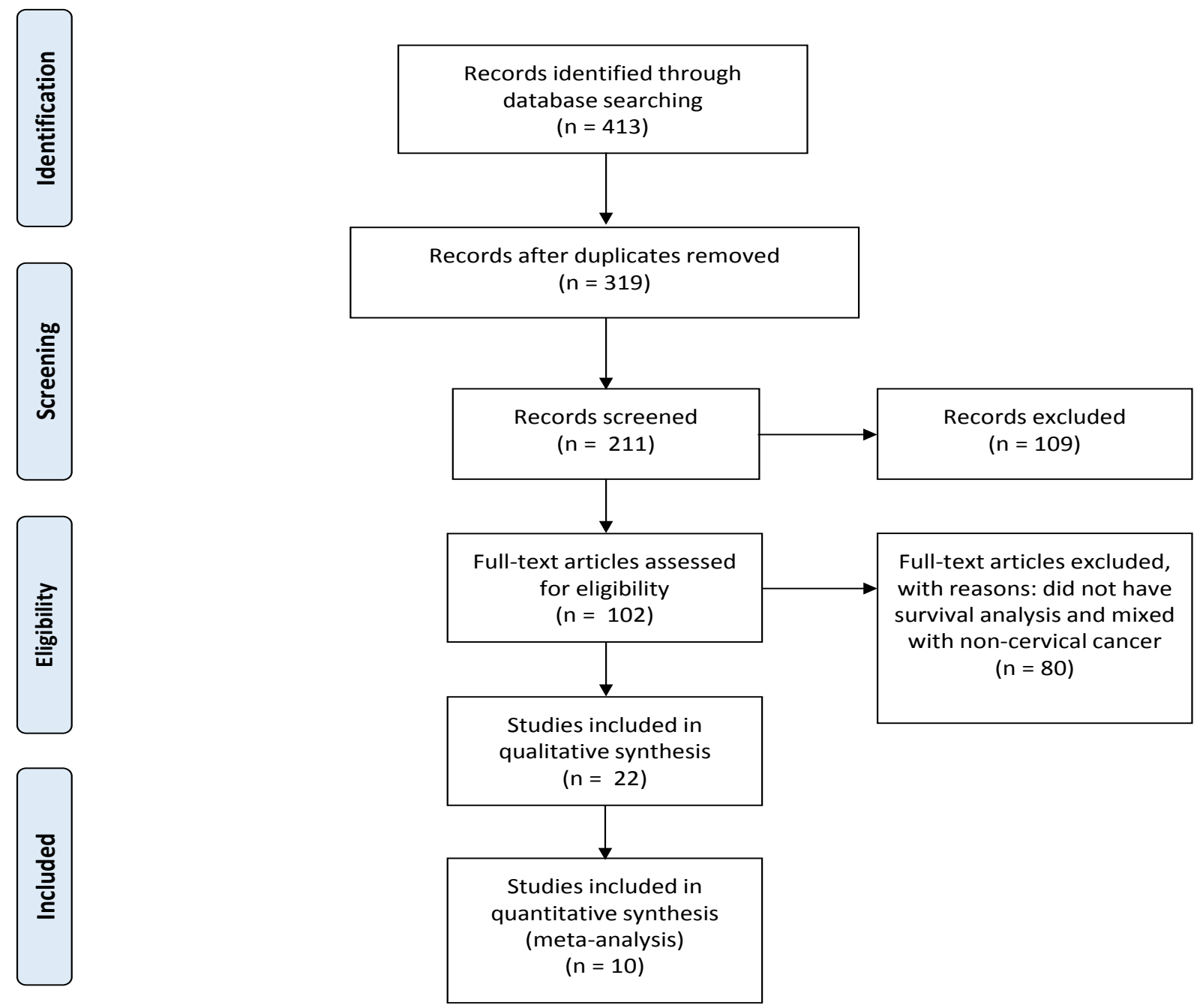

Figure 1. Figure 1. PRISMA Flow Diagram (Moher et al., 2009). 


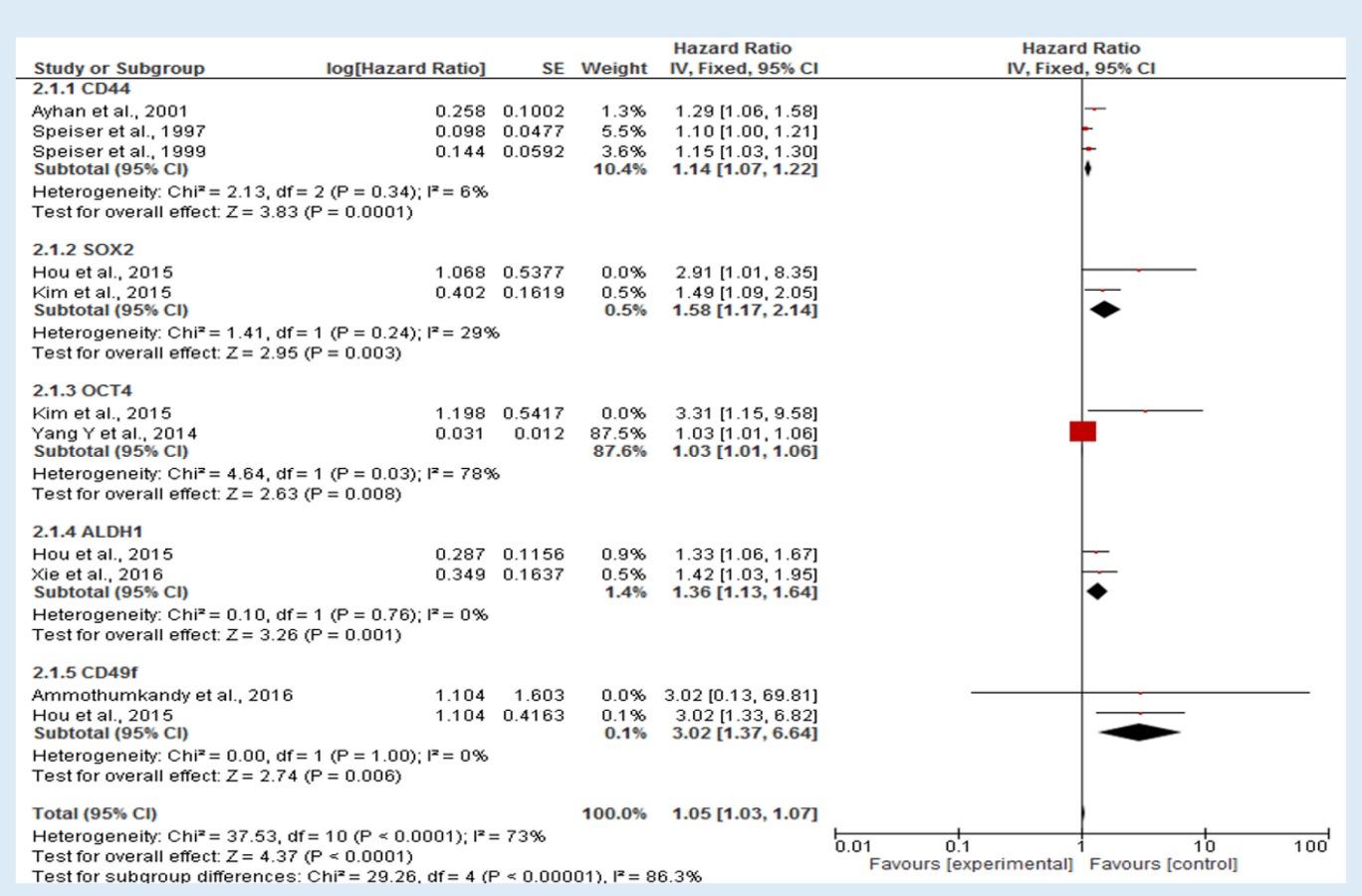

Figure 2. Forest Plot of the Association between Cancer Stem Cell Markers Expression and Overall Survival in Cervical Cancer Patients.

markers significantly predicted worse $\mathrm{OS}(\mathrm{HR}=1.05,95 \%$ CI: $1.03-1.07, \mathrm{P}<0.0001)$ with heterogenous studies $\left(\mathrm{I}^{2}=\right.$ $\left.73 \% . \mathrm{P}_{\mathrm{h}}<0.0001\right)$. A fixed-effect model was utilized. The correlations between individual CSC markers and OS were studied. The results showed that overexpression of CD44 (HR $=1.14,95 \%$ CI: $1.07-1.22$, P $0.0001, \mathrm{I}^{2}=$ $6 \%, \mathrm{Ph} 0.34), \mathrm{SOX} 2(\mathrm{HR}=1.58,95 \% \mathrm{CI}: 1.17-2.14$, P $0.003, \mathrm{I}^{2}=29 \%$, Ph 0.24), OCT4 (HR=1.03, 95\% CI: $\left.1.01-1.06, \mathrm{P} 0.008, \mathrm{I}^{2}=78 \%, \mathrm{Ph} 0.03\right), \mathrm{ALDH} 1(\mathrm{HR}=$ $1.36,95 \%$ CI: $\left.1.13-1.64, \mathrm{P} 0.001, \mathrm{I}^{2}=0 \%, \mathrm{Ph} 0.76\right)$ and CD49f $\left(\mathrm{HR}=3.02,95 \% \mathrm{CI}: 1.37-6.64, \mathrm{P} 0.006, \mathrm{I}^{2}=0 \%\right.$, $\mathrm{Ph} 1.00$ ) were associated with worse OS in cervical cancer patients. Another result from Hashiguchi et al. stated that CK17 was not associated with OS. Because there was only one study, we did not conduct the meta-analysis of this marker.

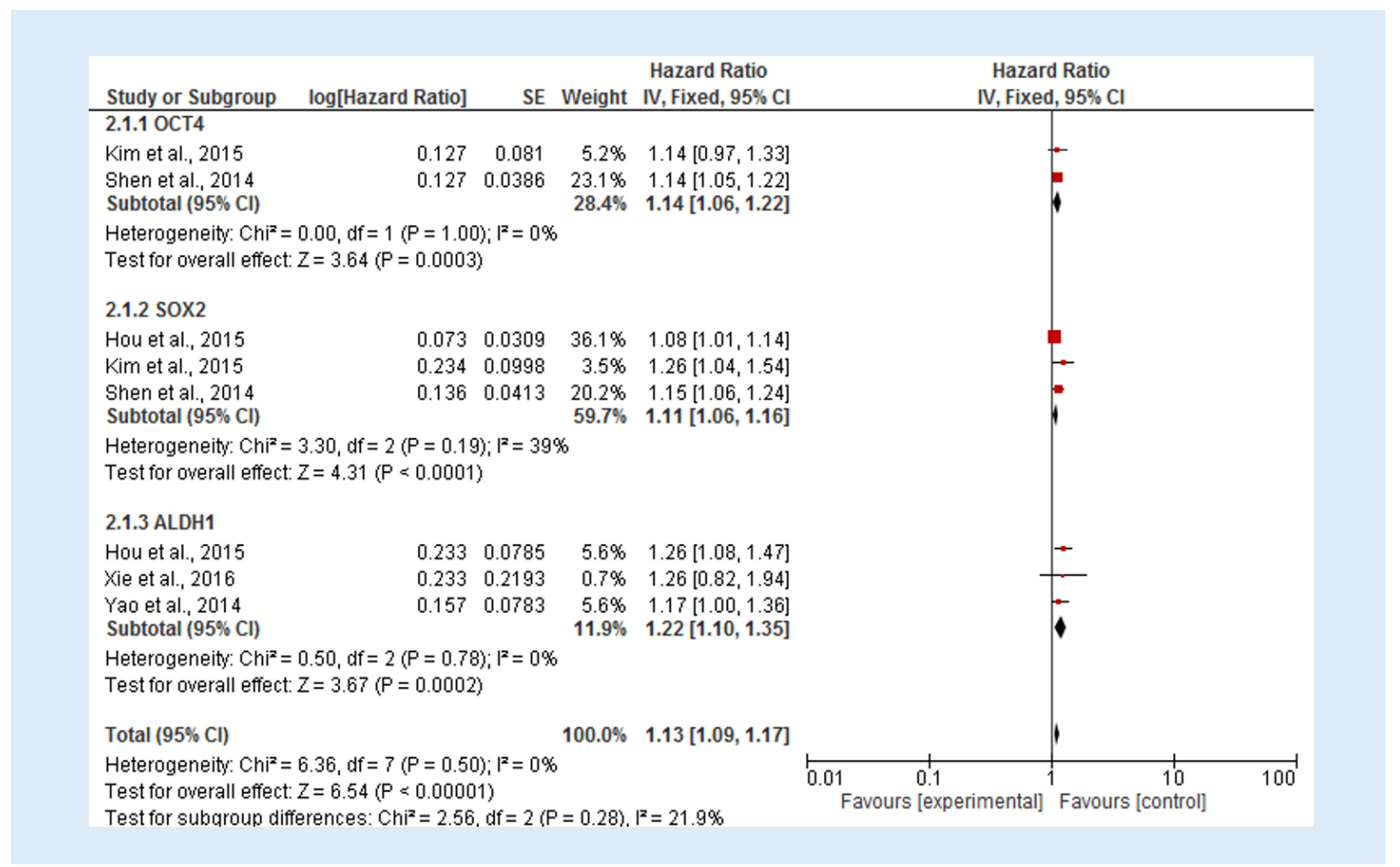

Figure 3. Forest Plot of The Correlation between Cancer Stem Cell (CSC) Markers Expression and Disease-Free Survival (DFS) in Cervical Cancer Patients. 


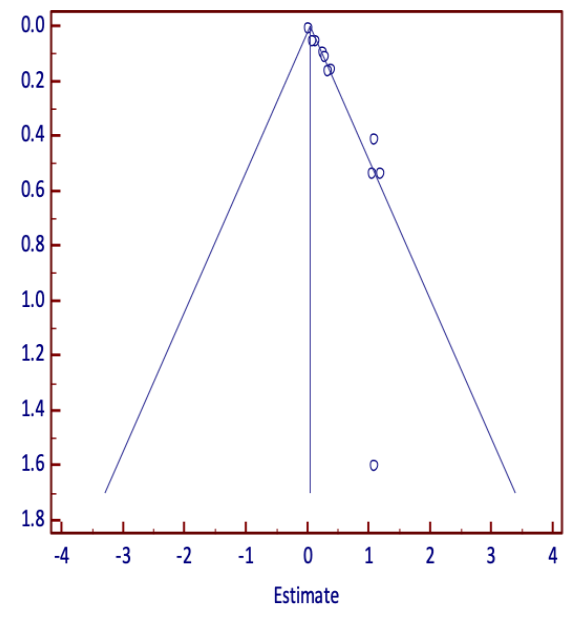

Overall OS (Egger's test $P<0.0001$, Begg's test $P$ 0.2714)

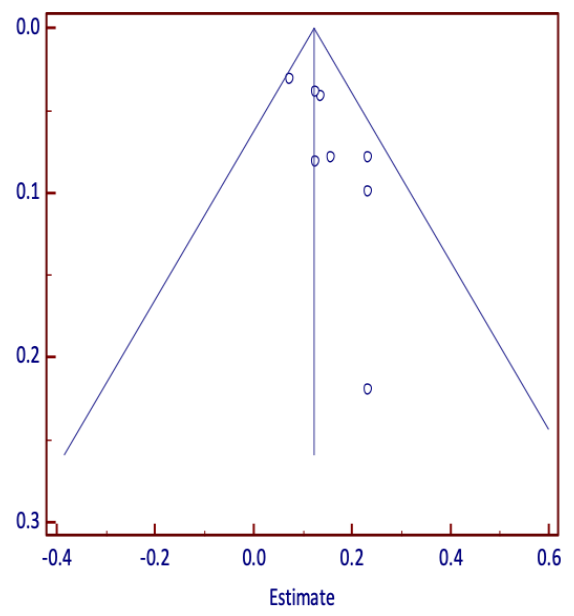

Overall DFS (Egger's test $P$ 0.0295, Begg's test $P$ 0.0833)

Figure 4. Funnel Plot to Examine Publication Bias for Overall Survival (OS) and Disease-Free Survival (DFS).

Association between cervical cancer stem cell (CCSC) marker expression and disease-free survival (DFS)

There were 5 studies, including 1,103 patients that investigated the association between the expression of CSC markers and DFS in cervical cancer patients. As shown in Figure 3, the overall pooled HR indicated a poor DFS in the cases with high expression of CCSC markers $(\mathrm{HR}=1.31,95 \% \mathrm{CI}: 1.09-1.17, \mathrm{P}<0.00001)$ with homogenous studies $\left(\mathrm{I}^{2}=0 \%, \mathrm{Ph} 0.50\right)$. Analyses for individual markers showed overexpression of CCSC markers, including OCT4 ( $\mathrm{HR}=1.14,95 \% \mathrm{CI}$ : $1.06-1.22$,
$\mathrm{P} 0.0003, \mathrm{I} 2=0 \%, \mathrm{Ph} 1.00), \mathrm{SOX} 2(\mathrm{HR}=1.11,95 \% \mathrm{CI}$ : $1.06-1.16, \mathrm{P}<0.0001, \mathrm{I} 2=39 \%, \mathrm{Ph} 0.19)$ and ALDH1 $(\mathrm{HR}=1.22,95 \% \mathrm{CI}: 1.10-1.35, \mathrm{P} 0.0002, \mathrm{I} 2=0 \%, \mathrm{Ph}$ 0.78 ) were associated significantly with poor DFS.

\section{Publication Bias}

As shown in Figure 4, 5, and 6, the Egger's and Begg's tests were used to evaluate potential bias. There were publication bias in the correlation of CCSC markers with overall OS and DFS. In subgroup analyses, the publication bias was not confirmed in the association of CCSC

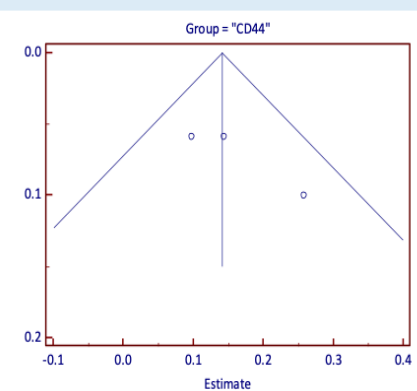

CD44 expression for OS (Egger's test $P<0.2615$, Begg's $P$ 0.2008)

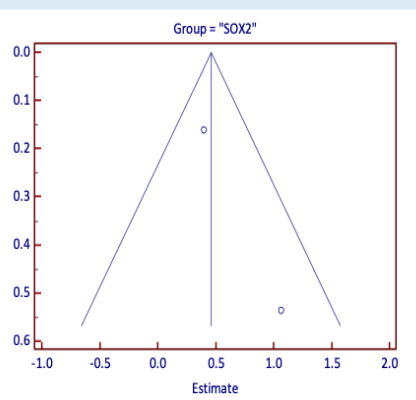

SOX2 expression for OS (Egger's test $P$ $<0.0001$, Begg's P 0.3173)

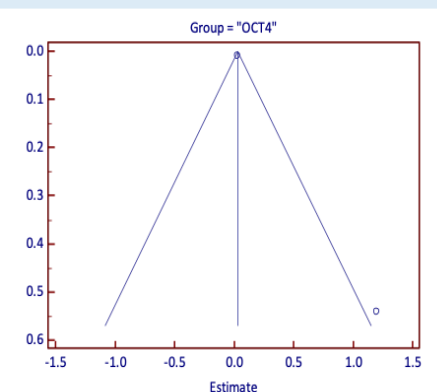

OCT4 expression for OS (Egger's test $\mathrm{P}$ $<0.0001$, Begg's P 0.3173)

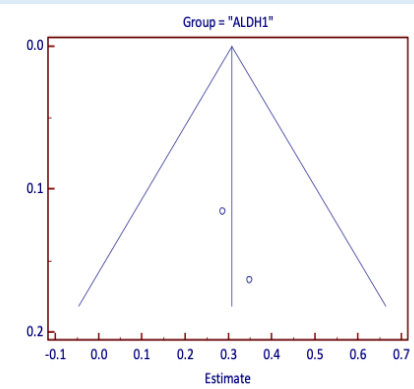

ALDH1 expression for OS (Egger's test $\mathrm{P}<0.0001$, Begg's $\mathrm{P} 0.3173$ )

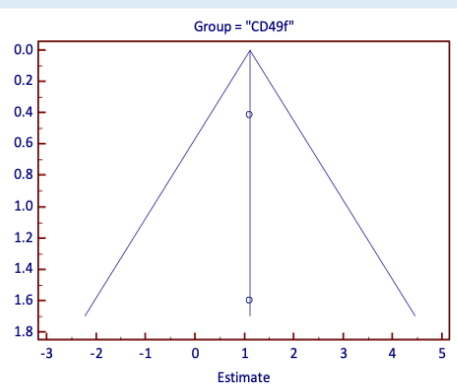

CD49f expression for OS (Egger's test $P$ $<0.0001$, Begg's test NA)

Figure 5. Funnel Plot to Examine Publication Bias of CD44, SOX2, OCT4, ALDH1, and CD49f for Overall Survival (OS). 


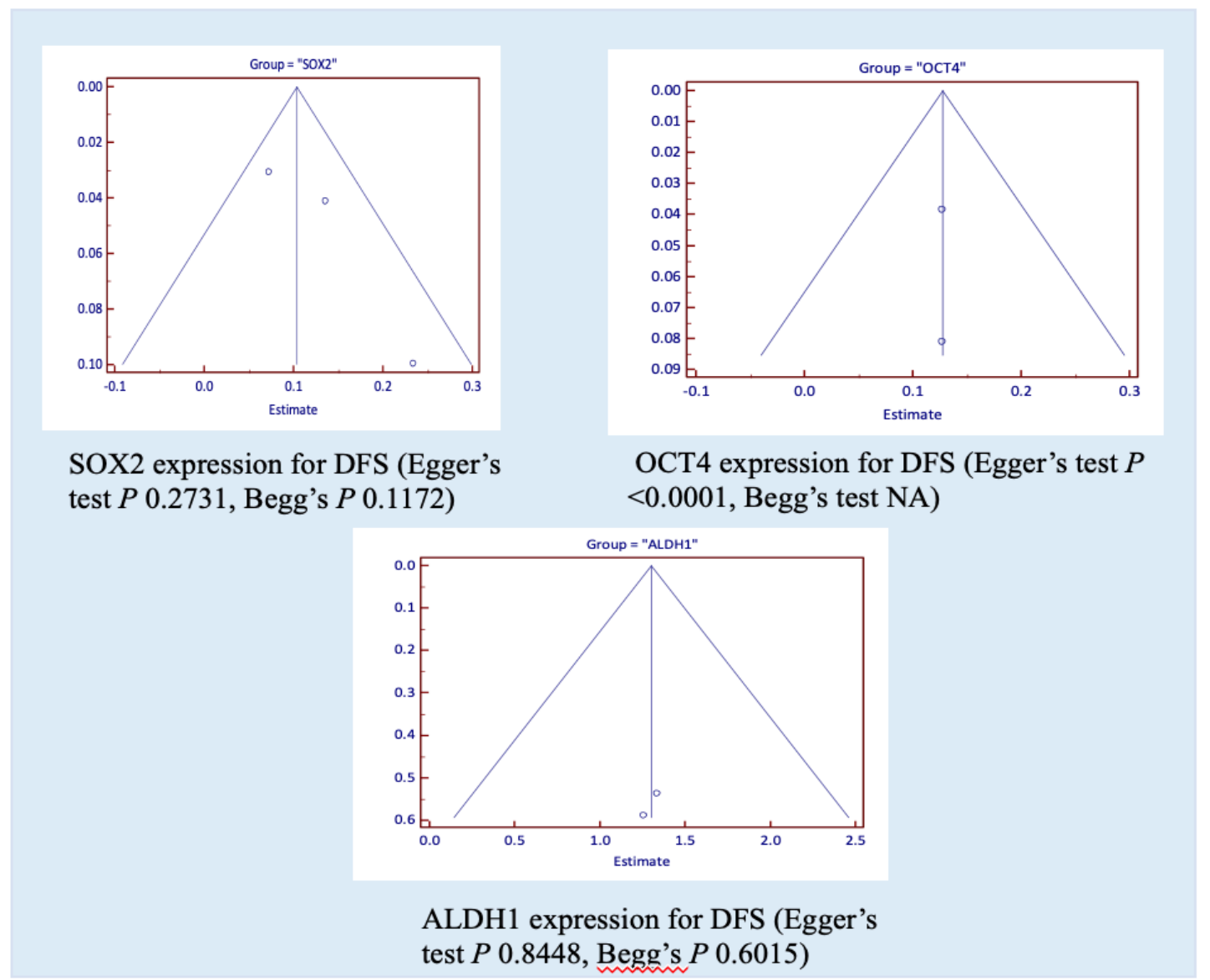

Figure 6. Funnel plot to examine publication bias of SOX2, OCT4, and ALDH1 for disease-free survival (DFS).

markers, including CD44 with OS; SOX2 and ALDH1 with DFS $(\mathrm{P}>0.05)$. There was some publication bias in the association of SOX2, OCT4, ALDH1, and CD49f with OS $(\mathrm{P}<0.05)$. In the DFS, the publication bias was confirmed in OCT4 analysis $(\mathrm{P}<0.05)$.

\section{Discussion}

In this study, we conducted a systematic review and meta-analysis of the CCSC markers to predict the OS and DFS. This review showed the most complete meta-analysis of CSC markers that have prognostic significances in cervical cancer. We found that high expressions of CD44, SOX2, OCT4, ALDH1, and CD49f were associated with poor OS. Meanwhile, overexpression of OCT4, SOX2, and ALDH1 were also associated with worse DFS. These findings suggest that CCSC markers expression may assist the clinicians in the management or assessment of the cervical cancer status after surgery.

The role of stem cells have been investigated in many cancers, including cervical cancer stem cells (CCSCs). Cancer stem cells (CSCs) have the abilities of pluripotency, to differentiate into a variety of cell types, and self-renewal. Some factors considered had the stemness character in a cancer cell, which contributed to carcinogenesis (Ji et al., 2014). Organista et al. reviewed that some studies were describing the phenotype of CCSCs, including MSI1, ALDH1, OCT3/4, SOX2, and CD49f (Organista-Nava et al., 2019).

The CSCs response to the radiotherapy fraction was also different from non-CSCs. The volume of non-CSC may decrease in the presence of DNA damage caused by ionizing radiation. In fact, CSCs actually showed more active evidence of DNA damage after radiation. This update occurs via the ataxia-telangiectasia mutate (ATM) and checkpoint kinases-1 and -2 (Chk1 / Chk2). The existence of rapid repopulation during and after radiotherapy is an important reason for the failure of radiotherapy which can increase the recurrence rate and affect survival (Bao et al., 2006; Signore et al., 2013).

In cervical cancer, cells with high expression of SOX2 could form the tumorspheres and tumor-initiating capacity in cervical cancer (Liu et al., 2014). Hou et al. also found that SOX2 predicted the poor OS and RFS. 18\% of patients with high expression of SOX 2 had recurrent cancer. Meanwhile, only 3\% patients with low expression had recurrent cancer. It was implied that SOX2 has a role in cancer progression (Hou et al., 2015). In line with that study, we also found that overexpression of SOX2 can predict a poor DFS in cervical cancer patients.

CD44 is a major adhesion molecule in the extracellular matrix, which implies the wide biological process. These molecules have been investigated in many populations of CSCs. CD44 is also important for tumor progression and metastasis (Castelli et al., 2017). CD44 plays an important role in stem cell communication with the microenvironment (Yan et al., 2015). High expression of CD44 in some solid tumors has been associated with a more aggressive type (Linge et al., 2016).

In hepatocellular carcinoma, the role of CD44 
expression is mediated by TGF- $\beta$ and correlated with poor prognosis (Mima et al., 2012). In cervical cancer cell line studies, cells with CD44-positive show a greater capacity for self-renewal (López et al., 2012; Ortiz-Sánchez et al., 2016). There is a theory that CD44 expression is activated via NOTCH pathways. These pathways affected cell proliferation, cell fate, differentiation, and cell death (Okamoto et al., 2001). We also found that CD44 overexpression predicted poor OS.

Sex determining region Y-box 2 (SOX2) has an essential function in many kinds of tissues and organs during embryonic development (Liu et al., 2014). This gene is also known to increase tumor proliferation (Yang $\mathrm{Z}$ et al., 2014). Some studies proved that SOX2 expressed in cervical cancer (Hou et al., 2015; Shen et al., 2014; Stewart and Crook, 2016). SOX2 maintained its characteristic, "stem cell-like properties" in cervical cancer cells which interacted with other stem cells, including Nanog and OCT3/4 (Boumahdi et al., 2014; Kim et al., 2015; K. Liu et al., 2013). There are four basic principles of radiotherapy, including repair, redistribution, repopulation, and reoxygenation. Expression of SOX2 was also associated with radio-resistance in squamous cervical cancer (SCC). Tumor cells which express SOX2 have a high capacity for self-renewal, differentiation, and tumor formation (Diehn et al., 2009).

Octamer-binding transcription factor 4 (OCT4) is defined as a group of genes which have pluripotential characteristics and self-renewal in CSC. OCT4 expression might take a role in carcinogenesis in cancers. Its expression is also associated with radiotherapy and chemotherapy resistance (Shen et al., 2014). The role of this gene could not be separated from other gene complexes, such as SOX2, Nanog, and ALDH1 (Organista-Nava et al., 2019). From our meta-analysis, high expression of OCT4 also can predict the poor OS and DFS. The recurrent activity was related to residual tumors after resection (Kim et al., 2015). Because of limited studies that assessed OCT4 (only two studies), further research is needed to clarify this result.

In our meta-analysis, we found that ALDH1 was significantly correlated with poor OS and DFS. This enzyme could reduce the reactive oxygen species (ROS) and reactive aldehyde. ALDH1 could support tumor development for carcinogenesis and the pluripotential effects in cervical cancer (Tomita et al., 2016). Therefore, high ALDH1 expression will affect the recurrence rate and poor survival.

Aldehyde dehydrogenase 1 (ALDH1) is an enzyme that contributes to aldehyde metabolism to reduce oxidative stress (Organista-Nava et al., 2019). ALDH1 has roles in cell proliferation, differentiation, embryonic development, and apoptosis via retinoic acid receptors ( $\mathrm{Li}$ et al., 2014; Ueda et al., 2013). This enzyme is involved in the early differentiation of stem cells by the oxidation of retinol into retinoic acid (Lohberger et al., 2012) This enzyme was correlated with malignancy, self-renewal of the tumor cell, and tumorigenesis in cervical cancer (Rao et al., 2012). Some studies have identified ALDH1 as a CSC marker in cervical cancer (Rusuldi and Askandar, 2017; Yao et al., 2011). ALDH also contributed to many pathways of carcinogenesis and stem cell signaling (Clark and Palle, 2016).

In cervical cancer therapy, ALDH expression is associated with the presence of chemo- and radioresistance. According to Hou et al., ALDH can predict poor survival in patients with cervical squamous cell carcinoma who have received adjuvant chemotherapy after surgery (Hou et al., 2015). Various studies have shown evidence of an association between ALDH expression, chemoresistance, and poor clinical outcomes in patients with cervical cancer (Organista-Nava et al., 2019). Another study result by Lv et al. found a total of $48.72 \%$ of patients showed lower ALDH1 expression before receiving chemoradiotherapy (Lv et al., 2015). ALDH is a cytosol enzyme that can detoxify cisplatin aquated so that the effectiveness of cisplatin therapy reduces cisplatin activity. When aquated cisplatin enters and forms cisplatin-DNA adduct, it is seen as a damage so that CSC releases its ability to carry out self-renewal through Chk1/2 kinase (DNA repair) (Rusuldi and Askandar, 2017).

Cluster differentiation 49f (CD49f) has been used to identify cervical cancer (López et al., 2012). Torimura et al. reported that CD49f plays a critical role in cancer stem cell maintenance and attachment of tumor cell to the laminin in hepatoma. They found that high expression of CD49f was associated with poor survival (Torimura et al., 1999). Study from Hou et al. also found that low expression of CD49f in cervical cancer correlated with poor OS and progression-free survival (PFS) (Hou et al., 2015). Expression of CD49f might be decreased in invasive areas of cancer tissue. It was also reported for other cell adhesion molecules (Ngan et al., 2007).

Based on this systematic review, there were some publication bias and homogeneous studies. The publication bias was not confirmed only in the association of CD44 with OS and SOX2 with DFS. The heterogeneous study was only confirmed in the association of OCT4 with OS. But, there was no publication bias in individual study. The cut-off of CCSC markers varied due to many factors, including different antibodies used to detect each marker, different participant and specimen characteristics. The study quality in this meta-analysis also did not represent The study quality in this meta-analysis also did not represent a high-quality score.

In conclusion, OCT4, SOX2, and ALDH1 might have potential roles as prognostic biomarkers in cervical cancer. Further research with a multicenter, high-quality study, and larger sample sizes should be conducted to clarify the results.

\section{Author Contribution Statement}

MNF and FK conceived and designed the study; MNF, INH and FK searched the database, screened titles, abstracts, and full text studies included. MNF, INH, and FK performed study quality assessments, data analyses, and data interpretations. MNF and INH drafted the initial manuscript. MNF, INH, and FK performed critical revisions of the manuscript and finalized the manuscript. Then, all authors approved the final version of the manuscript.

Asian Pacific Journal of Cancer Prevention, Vol 22 


\section{Acknowledgments}

The authors thank the Gynecology Oncology Division, Department of Obstetrics and Gynecology, Universitas Indonesia, Dr. Cipto Mangunkusumo General Hospital, Faculty of Medicine, Jakarta, Indonesia and the Faculty of Medicine, Public Health and Nursing Universitas Gadjah Mada, Prof. dr. Moh. Hakimi, Sp.OG(K)., Ph.D for help by supporting this review. Dewi Masitoh for technical supporting during the process of this study.

\section{Funding Statement}

This study received no specific grant from any funding agency.

\section{Ethical Approval}

The data was synthesized from the published research (secondary data analysis). So, this study did not need ethical approval. This study was registered at www.crd. york.ac.uk (PROSPERO-CRD42021237072) and not part of the approved student thesis. There was no availability data in this study.

\section{Conflict of Interest}

There was no potential conflict of interest.

\section{References}

American Cancer Society (2021). Key Statistics for Cervical Cancer. Available from: https://www.cancer.org/cancer/ cervical-cancer/about/key-statistics.html [Accessed on 26 January 2021].

Bao S, Wu Q, McLendon RE, et al (2006). Glioma stem cells promote radioresistance by preferential activation of the DNA damage response. Nature, 444, 756-60.

Boumahdi S, Driessens G, Lapouge G, et al (2014). SOX2 controls tumour initiation and cancer stem-cell functions in squamous-cell carcinoma. Nature, 511, 246-50.

Castelli G, Pelosi E, Testa U (2017). Liver cancer: olecular characterization, clonal evolution and cancer stem cells. Cancers, 9, 1-36.

Clark DW, Palle K (2016). Aldehyde dehydrogenases in cancer stem cells: potential as therapeutic targets. Ann Transl Med, 4, 1-8.

Dalerba P, Cho RW, Clarke MF (2007). Cancer stem cells: models and concepts. Annu Rev Med, 58, 267-84.

Diehn M, Cho RW, Lobo NA, et al (2009). Association of reactive oxygen species levels and radioresistance in cancer stem cells. Nature, 458, 780-3.

Dobbin ZC, Landen CN (2013). Isolation and characterization of potential cancer stem cells from solid human tumors:potential applications. Curr Protoc Pharmacol, 63, 1-24.

Hashiguchi M, Masuda M, Kai K et al (2019). Decreased cytokeratin 7 expression correlates with the progression of cervical squamous cell carcinoma and poor patient outcomes. J Obstet Gynaecol Res, 45, 2228-36.

Hou T, Zhang W, Tong C, et al (2015). Putative stem cell markers in cervical squamous cell carcinoma are correlated with poor clinical outcome. BMC Cancer, 15, 1-8.

Ji J, Wei X, Wang Y (2014). Embryonic stem cell markers SOX-2 and OCT4 expression and their correlation with WNT signal pathway in cervical squamous cell carcinoma. Int J Clin Exp Pathol, 7, 2470-6.
Kim BW, Cho H, Choi CH, et al (2015). Clinical significance of OCT4 and SOX2 protein expression in cervical cancer. BMC Cancer, 15, 1-8.

Li J, Feng ZC, Yeung FS-H, et al (2014). Aldehyde dehydrogenase 1 activity in the developing human pancreas modulates retinoic acid signalling in mediating islet differentiation and survival. Diabetologia, 57, 754-64.

Linge A, Lohaus F, Löck S, et al (2016). HPV status, cancer stem cell marker expression, hypoxia gene signatures and tumour volume identify good prognosis subgroups in patients with HNSCC after primary radiochemotherapy: a multicentre retrospective study of the German Cancer Consortium Radiation. Radiother Oncol, 121, 1-10.

Liu H, Wang Y-J, Bian L, et al (2016). CD44+/CD24+ cervical cancer cells resist radiotherapy and exhibit properties of cancer stem cells. Eur Rev Med Pharmacol Sci, 20, 1745-54.

Liu K, Lin B, Zhao M, et al (2014). The multiple roles for SOX2 in stem cell maintenance and tumorigenesis. Cell Signal, 25, 1-19.

Liu X-F, Yang W-T, Xu R, Liu J-T, Zheng P-S (2014). Cervical cancer cells with positive SOX2 expression exhibit the properties of cancer stem cells. PLoS One, 9, 1-12.

Lohberger B, Rinner B, Stuendl N, et al (2012). Aldehyde dehydrogenase 1, a potential marker for cancer stem cells in human sarcoma. PLoS One, 7.

López J, Poitevin A, Mendoza-Martínez V, Pérez-Plasencia C, García-Carrancá A (2012). Cancer-initiating cells derived from established cervical cell lines exhibit stem-cell markers and increased radioresistance. BMC Cancer, 28, 1-14.

Lv Y, Yang L, Wang F (2015). Chemoradiation therapy reduces aldehyde dehydrogenase 1 expression in cervical cancer but does not improve patient survival. Med Oncol, 32, 1-6.

Mima K, Okabe H, Ishimoto T, et al (2012). CD44s regulates the TGF- $\beta$-mediated mesenchymal phenotype and is associated with poor prognosis in patients with hepatocellular carcinoma. Cancer Res, 72, 3414-23.

Moher D, Liberati A, Tetzlaff J, et al (2009). Preferred reporting items for systematic reviews and meta-analyses: The PRISMA statement. $B M J, 6,1-6$.

Muralikrishnan V, Hurley TD, Nephew KP (2020). Targeting aldehyde dehydrogenases to eliminate cancer stem cells in gynecologic malignancies. Cancers, 12, 1-20.

Ngan CY, Yamamoto H, Seshimo I, et al (2007). A multivariate analysis of adhesion molecules expression in assessment of colorectal cancer. J Surg Oncol, 95, 652-62.

Okamoto I, Kawano Y, Murakami D, et al (2001). Proteolytic release of CD44 intracellular domain and its role in the CD44 signaling pathway. J Cell Biol, 155, 755-62.

Organista-Nava J, Gómez-Gómez Y, Garibay-Cerdenares OL, Leyva-Vázquez MA, Illades-Aguiar B (2019). Cervical cancer stem cell-associated genes: prognostic implications in cervical cancer. Oncol Lett, 18, 7-14.

Ortiz-Sánchez E, Santiago-López L, Cruz-Domínguez VB, et al (2016). Characterization of cervical cancer stem cell-like cells: phenotyping, stemness, and human papilloma virus co-receptor expression. Oncotarget, 31, 31943-54.

Pimple S, Mishra G, Shastri S (2018). Global strategies for cervical cancer prevention. Curr Opin Obstet Gynecol, 28, 4-10.

Rao Q-X, Yao T-T, Zhang B-Z, et al (2014). Expression and functional role of ALDH1 in cervical carcinoma cells. Asian Pac J Cancer Prev, 13, 1325-31.

Rasjidi I (2009). Epidemiologi kanker serviks. Indones J Cancer, 3, 103-8.

Rusuldi G, Askandar B (2017). Ekspresi CD44 dan ALDH1 (Penanda Sel Punca Kanker) sebagai Prediktor Respons Kemoterapi Neoadjuvant Cisplatin pada Kanker Serviks 
Uteri Stadium IIB. Indones J Cancer, 11, 87-95.

Shen L, Huang X, Xie X, et al (2014). High expression of SOX2 and OCT4 indicates radiation resistance and an independent negative prognosis in cervical squamous Cell carcinoma. J Histochem Cytochem, 62, 499-509.

Signore M, Ricci-Vitiani L, De Maria R (2013). Targeting apoptosis pathways in cancer stem cells. Cancer Lett, 332, 374-82.

Stewart CJR, Crook M (2016). SOX2 expression in cervical intraepithelial neoplasia grade 3 (CIN3) and superficially invasive (Stage IA1) squamous carcinoma of the cervix. Int J Gynecol Pathol, 35, 566-73.

Tomita H, Tanaka K, Hara A (2016). Aldehyde dehydrogenase 1A1 in stem cells and cancer. Oncotarget, 7, 11018-32.

Torimura T, Ueno T, Kin M, et al (1999). Integrin $\alpha 6 \beta 1$ plays a significant role in the attachment of hepatoma cells to laminin. J Hepatol, 31, 1-8.

Ueda K, Ogasawara S, Akiba J, et al (2013). Aldehyde dehydrogenase 1 identifies cells with cancer stem cell-like properties in a human renal cell carcinoma cell line. PLoS One, 8.

Yan Y, Zuo X, Wei D (2015). Concise review: emerging role of CD44 in cancer stem cells: a promising biomarker and therapeutic target. Stem Cells Transl Med, 4, 1033-43.

Yang Y, Wang Y, Yin C, Li X (2014). Clinical significance of the stem cell gene Oct-4 in cervical cancer. Tumor Biol, 35, 5339-45.

Yao T, Chen Q, Zhang B, Zhou H, Lin Z (2011). The expression of ALDH1 in cervical carcinoma. Med Sci Monit, 17, 1-5.

Yoon HI, Cha J, Keum KC, et al (2015). Treatment outcomes of extended-field radiation therapy and the effect of concurrent chemotherapy on uterine cervical cancer with para-aortic lymph node metastasis. Radiat Oncol, 13, 1-10.

\section{c) (7) @}

This work is licensed under a Creative Commons AttributionNon Commercial 4.0 International License. 